\title{
Stability of Solitary Waves for a Generalized Nonlinear Coupled Schrodinger Systems
}

\section{Orlando Lopes}

Instituto de Matemática e Estatística, Universidade de São Paulo Rua do Matão, 1010, Caixa postal 66281, CEP 05315-970, São Paulo, SP, Brasil

E-mail address: olopes@ime.usp.br

\begin{abstract}
In this paper we show that the standing waves of the form $\left(e^{i \beta t} u(x), e^{i \beta t} u(x)\right), \beta>0, u(x)$ real and positive, are stable for the system

$$
\begin{aligned}
& i \frac{\partial u}{\partial t}+u_{x x}+\left(|u|^{2 p-2}+\gamma|v|^{p}|u|^{p-2}\right) u=0 \\
& i \frac{\partial v}{\partial t}+v_{x x}+\left(\gamma|u|^{p}|v|^{p-2}+|v|^{2 p-2}\right) v=0
\end{aligned}
$$$$
\text { provided } 2 \leq p<3 \text { and } 0<\gamma \neq p-1 \text {. }
$$

The Morse index of such solution is one for $\gamma>p-1$ and two for $0<\gamma<p-1$ but it is stable in both cases.
\end{abstract}

\section{Introduction and Statement of the Result}

The existence and stability of standing waves of the form $e^{i \beta t} u(x), \beta>0$, $u(x)$ real and positive, for the equation

$$
i \frac{\partial u}{\partial t}+\Delta u+|u|^{q} u=0
$$

are very well understood. For instance, the positive standing waves are stable provided $0<q<4 / N$. Moreover, for $q \geq 4 / N$ blow-up results are available. More recently, systems of the following form have also been considered

$$
\begin{aligned}
& i \frac{\partial u}{\partial t}+\Delta u+\left(|u|^{2}+\gamma|v|^{2}\right) u=0 \\
& i \frac{\partial v}{\partial t}+\Delta v+\left(\gamma|u|^{2}+|v|^{2}\right) v=0
\end{aligned}
$$

where $u$ and $v$ belong to $\mathbb{C}$ and $x$ belong to $\mathbb{R}^{N} . N$ will be 1,2 or 3 . Due to its application in Nonlinear Optics, system (1.2) has called the attention of 
many researchers in both pure and applied areas (see [8] for a partial list of references).

Here in this paper we consider the following generalized version of (1.2)

$$
\begin{aligned}
& i \frac{\partial u}{\partial t}+\Delta u+\left(|u|^{2 p-2}+\gamma|v|^{p}|u|^{p-2}\right) u=0 \\
& i \frac{\partial v}{\partial t}+\Delta v+\left(\gamma|u|^{p}|v|^{p-2}+|v|^{2 p-2}\right) v=0 .
\end{aligned}
$$

At least formally, system (1.3) has the following three complex first integrals:

$$
\begin{aligned}
E(u, v) & =\frac{1}{2} \int_{\mathbb{R}^{N}}\left(|\operatorname{grad} u(x)|^{2}+|\operatorname{grad} v(x)|^{2}\right) \\
& -\int_{\mathbb{R}^{N}}\left(\frac{1}{2 p}|u(x)|^{2 p}+\frac{\gamma}{p}|u(x)|^{p}|v(x)|^{p}+\frac{1}{2 p}|v(x)|^{2 p}\right) d x \\
Q_{1}(u) & =\frac{1}{2} \int_{\mathbb{R}^{N}}|u(x)|^{2} d x \quad Q_{2}(v)=\frac{1}{2} \int_{\mathbb{R}^{N}}|v(x)|^{2} d x
\end{aligned}
$$

Standing waves are solutions of (1.3) of the form $\left(e^{i \beta_{1} t} u(x), e^{i \beta_{2} t} v(x)\right)$ where $u(x)$ and $v(x)$ are real and $\beta_{i}$ are real and positive. Then $(u(x), v(x))$ solves the elliptic system

$$
\begin{aligned}
-\Delta u+\beta_{1} u-\left(|u|^{2 p-2}+\gamma|v|^{p}|u|^{p-2}\right) u & =0 \\
-\Delta v+\beta_{2} v-\left(\gamma|u|^{p}|v|^{p-2}+|v|^{2 p-2}\right) v & =0
\end{aligned}
$$

The solutions of (1.6) are critical points of the real energy

$$
\begin{aligned}
E(u, v)=\frac{1}{2} \int_{\mathbb{R}^{N}}\left(|\operatorname{grad} u(x)|^{2}+|\operatorname{grad} v(x)|^{2}\right) d x & \\
& -\int_{\mathbb{R}^{N}}\left(\frac{1}{2 p}|u|^{2 p}+\frac{\gamma}{p}|u|^{p}|v|^{p}+\frac{1}{2 p}|v|^{2 p}\right) d x
\end{aligned}
$$

under the constraints

$$
\int_{\mathbb{R}^{N}} u^{2}(x) d x=c_{1} \quad \int_{\mathbb{R}^{N}} v^{2}(x) d x=c_{2} .
$$

For $1<p<N /(N-2)$ if $N \geq 3$ and $p>1$ for $N=1,2$, the existence of positive solutions of (1.6) has been proved in [6] generalizing results obtained previously in [1], [2] and [4],[5], among others, for the case $p=2$. 
Since existence of standing waves is established, the next thing is to study their stability. The three complex functionals (1.4-1.5) are invariant under the gauge and the translation transformations

$$
(u, v) \longrightarrow\left(e^{i \theta} u(\cdot+c), e^{i \psi} v(\cdot+c)\right) \quad \theta, \psi \in \mathbb{R}, c \in \mathbb{R}^{N} .
$$

The Cauchy problem for (1.3) is well posed in the complex space $H^{1}\left(\mathbb{R}^{N}\right) \times H^{1}\left(\mathbb{R}^{N}\right)$ and, as usual, the concept of stability is taken with respect with the norm of that space.

Definition. The standing wave $\left(e^{i \beta_{1} t} u(x), e^{i \beta_{2} t} v(x)\right)$ is orbitally stable with respect to system (1.3) if for any $\epsilon>0$ there is a $\delta>0$ such that if $(\tilde{u}(t, x), \tilde{v}(t, x)) \quad$ is a solution of (1.3) satisfying $\|(\tilde{u}(0, \cdot,) \tilde{v}(0 \cdot \cdot))-(u(\cdot), v(\cdot))\|<\delta$ then $\inf \|\left(e^{i \theta} \tilde{u}(t, \cdot+c), e^{i \psi} \tilde{v}(\cdot+c)\right)-$ $(u(\cdot), v(\cdot)) \|<\epsilon$, for all $t \in \mathbb{R}$, where the infimum is taken over $\theta, \psi \in$ $\mathbb{R}, c \in \mathbb{R}^{N}$.

System (1.3) has standing waves of the form (same frequency) $\left(e^{i \beta t} u(x), e^{i \beta t} u(x)\right), \beta>0$, provided $u$ solves

$$
-\Delta u+\beta u-(\gamma+1) u^{2 p-1}=0 .
$$

Our main result is the following:

Theorem I.1. For $N=1,0<\gamma \neq p-1, \beta>0$ and $2 \leq p<3$ the standing wave $\left(e^{i \beta t} u(x), e^{i \beta t} u(x)\right)$, where $u \in H^{1}(\mathbb{R})$, is a real positive solution of (1.10), is stable.

\section{Remarks}

1) In the case $p=2$, theorem 1.1 has been proved in [7] but some arguments do not to work for more general powers. Notice that the range of the power $p$ for which stability holds, is the same as for the single equation (1.1). However, as we will explain later in this case, the proof of the stability for the system is more difficult.

2) If $N=2$ and $1<p<2$, parts of the proof of the stability also works. But in that case, some terms appearing for instance in (2.12) below, are not differentiable. A similar problem arises for $N=3$.

Section two is devoted to the proof of theorem 1.1. A careful qualitative spectral analysis is required to show that all conditions to use the method presented in [3] are satisfied.

\section{Proof of the main result}

We consider a positive solution $\left(u_{\beta_{1}, \beta_{2}}(x), v_{\beta_{1}, \beta_{2}}(x)\right)$ of (1.6) depending smoothly on the parameters $\beta_{1}, \beta_{2}$. As we will see later, such family exists for $\left(\beta_{1}, \beta_{2}\right)$ close to the diagonal. From now on, to simplify the notation, 
we drop the subscript $\left(\beta_{1}, \beta_{2}\right)$ and that solution will be denoted simply by $(u(x), v(x))$. We define the quantities:

$$
Q_{1}\left(\beta_{1}, \beta_{2}\right)=\frac{1}{2} \int_{\mathbb{R}^{N}} u^{2}(x) d x \quad Q_{2}\left(\beta_{1}, \beta_{2}\right)=\frac{1}{2} \int_{\mathbb{R}^{N}} v^{2}(x) d x
$$

and the self adjoint operators

$$
\begin{gathered}
L_{1} h=-h_{x x}+\beta_{1} h-\left(u^{2 p-2}+\gamma v^{p} u^{p-2}\right) h \\
L_{2} k=-k_{x x}+\beta_{2} k-\left(\gamma u^{p} v^{p-2}+v^{2 p-2}\right) k \\
L(h, k)=\left(-h_{x x}+\beta_{1} h-\left((2 p-1) u^{2 p-2}+\gamma(p-1) u^{p-2} v^{p}\right) h-\gamma p v^{p-1} u^{p-1} k,\right. \\
-k_{x x}+\beta_{2} k-\gamma p u^{p-1} v^{p-1} h-\left((2 p-1) v^{2 p-2}+\gamma(p-1) u^{p} v^{p-2}\right) k
\end{gathered}
$$

A standing wave is stable if it is a local minimizer of the complex energy $E(u, v)$ defined by (1.4) subject to the two constraints $Q_{1}=c_{1}, Q_{2}=c_{2}$, where $Q_{1}$ and $Q_{2}$ are given by (1.5). A very general method for stability is presented in [3] and, according to it, for a given $\left(\beta_{1}, \beta_{2}\right)$, the standing wave $(u, v)$ is orbitally stable if the three following conditions are satisfied:

C1: zero is a simple eigenvalue of $L_{1}$ and of $L_{2}$ with eigenfunctions $u$ and $v$ respectively, and all the other eigenvalues are positive;

$\mathrm{C} 2$ : the kernel of $L$ is spanned by $\left(\frac{\partial u}{\partial x}, \frac{\partial v}{\partial x}\right)$;

C3. the number of positive eigenvalues of the symmetric $2 \times 2$ symmetric matrix

$$
R\left(\beta_{1}, \beta_{2}\right)=\left(\frac{\partial Q_{i}\left(\beta_{1}, \beta_{2}\right)}{\partial \beta_{j}}\right) \quad i, j=1,2
$$

is equal to the number of negative eigenvalues of $L$ defined by (2.14).

The fact that $u$ and $v$ are eigenfunctions of $L_{1}$ and $L_{2}$, respectively, associated to the zero eigenvalue follows from 1.6) (gauge invariance). Similarly, the fact that $\left(\frac{\partial u}{\partial x}, \frac{\partial v}{\partial x}\right)$ are eigenfunctions of $L$ associated to the zero eigenvalue follows from differentiation of (1.6) with respect to $x$ (translation invariance.) Therefore, conditions $\mathrm{C} 1$ and $\mathrm{C} 2$ say that standing wave $(u, v)$ is non degenerate in the sense that the multiplicity of zero as an eigenvalue of the full (complex) linearized operator is equal to the dimension of the invariance group.

Moreover, the fact that zero is a simple eigenvalue of the operators $L_{1}$ and $L_{2}$ follows easily because $u$ and $v$ are eigenfunctions of $L_{1}$ and $L_{2}$ associated to the zero eigenvalue. Since they are positive (we are assuming that 
since the beginning), they are the principal eigenfunctions and, hence, zero is a simple eigenvalue of them and all the other eigenvalues are positive. This means that condition $\mathrm{C} 1$ is verified for all values of $\beta_{1}$ and $\beta_{2}$. The verification of $\mathrm{C} 2$ and $\mathrm{C} 3$ is much more difficult and, as stated in theorem 1.1, we will do it in a particular case $\beta_{1}=\beta_{2}$ only. If the quantities $Q_{i}\left(\beta_{1}, \beta_{2}\right), i=1,2$ were known explicitly in terms of $\beta_{1}$ and $\beta_{2}$, the verification of condition $\mathrm{C} 2$ would be easy. For instance, in the case of the single Schrodinger equation (1.1) (and a single frequency $\beta$ ), using a scaling argument we can calculate $Q(\beta)$ for all values of $\beta$. In the case treated here, $Q_{1}\left(\beta_{1}, \beta_{2}\right)$ and $Q_{2}\left(\beta_{1}, \beta_{2}\right)$ can be calculated at the diagonal $\beta_{1}=\beta_{2}=\beta$ only. However, even in the case $\beta_{1}=\beta_{2}=\beta$, in (2.15) $\beta_{1}$ and $\beta_{2}$ are independent variables and this brings some difficulty because there is no explicit formula for $Q_{i}\left(\beta_{1}, \beta_{2}\right)$ in a full neighborhood of the diagonal $(\beta, \beta)$.

As we have pointed out earlier in this paper, for $\beta_{1}=\beta_{2}=\beta$ and $N=1$, system (1.6) has a solution $(u, v)$ with $u=v$ provided $u$ solves the single equation

$$
-u_{x x}+\beta u-(\gamma+1) u^{2 p-1}=0 .
$$

We start with the following very well known result.

Lemma II.1. For $p>1$, there is a unique symmetric positive function $\phi_{0}(x)$ belonging to $H^{1}\left(\mathbb{R}^{N}\right)$ satisfying

$$
-\phi_{0}^{\prime \prime}+\phi_{0}-\phi_{0}^{2 p-1}=0 .
$$

Moreover, $\phi_{0}$ is smooth and tends to zero exponentially at infinity together with their derivatives. Furthermore, the linearized operator

$$
L_{0} h=-h^{\prime \prime}+h-(2 p-1) \phi_{0}^{2 p-2} h
$$

has exactly one negative eigenvalue and the kernel of $L_{0}$ is spanned by $\phi_{0}^{\prime}$.

In terms of $\phi_{0}$ given by lemma II.1, the solution $u$ of (2.16) is given by

$$
u_{\beta}(x)=\left(\frac{\beta}{\gamma+1}\right)^{1 /(2 p-2)} \phi_{0}(\sqrt{\beta} x) .
$$

To prove theorem I.1 we will verify conditions C2 and C3 above because we have already proved that condition $\mathrm{C} 1$ is satisfied for all values of $\beta_{1}$ and $\beta_{2}$. We start with the verification of condition C2.

For $\beta_{1}=\beta_{2}=\beta$ and $u=v$, the operator $L$ defined by (2.14) becomes

$$
\begin{aligned}
L(h, k)=( & -h_{x x}+\beta h-(2 p-1+\gamma(p-1)) u^{2 p-2} h-\gamma p u^{2 p-2} k, \\
& -k_{x x}+\beta k-\gamma p u^{2 p-2} h-\left(2 p-1+\gamma(p-1) u^{2 p-2} k\right) .
\end{aligned}
$$

Assuming

$$
-h_{x x}+\beta h-(2 p-1+\gamma(p-1)) u^{2 p-2} h-\gamma p u^{2 p-2} k=\lambda h
$$




$$
\left.-k_{x x}+\beta k-\gamma p u^{2 p-2} h-(2 p-1+\gamma(p-1)) u^{2 p-2} k\right)=\lambda k
$$

and defining $r=h+k$ and $q=h-k$ we see that (2.22-2.23) decouples into

$$
M_{1} r \widehat{=}-r_{x x}+\beta r-(2 p-1)(\gamma+1) u^{2 p-2} r=\lambda r
$$

and

$$
M_{2} q \widehat{=}-q_{x x}+\beta q+(\gamma+1-2 p) u^{2 p-2} q=\lambda q .
$$

Lemma II.2. The kernel of $M_{1}$ is spanned by $\frac{\partial u}{\partial x}$ and $M_{1}$ has exactly one negative eigenvalue.

Proof. Since $M_{1}$ is the linearization of (2.16), the lemma follows easily.

Lemma II.3. Define

$$
M_{\epsilon} s=-s_{x x}+s-\epsilon \phi_{0}^{2 p-2}(x) s .
$$

Then

i) for $\epsilon<1$ the operator $M_{\epsilon}$ is positive definite.

ii) for $1<\epsilon<2 p-1$ the operator $M_{\epsilon}$ is invertible and it has exactly one negative eigenvalue;

Proof. As a consequence of the variational caracterization of the point spectrum of a self adjoint operator, we see that the point spectrum of $M_{\epsilon}$ moves strictly to the left as $\epsilon$ increases. Moreover, due to (2.17), for $\epsilon=1$ the operator $M_{\epsilon}$ has $\phi_{0}$ as the principal eigenfunction corresponding to the zero eigenvalue. Furthermore, as a consequence of lemma II.1 concerning the operator $L_{0}$ defined by (2.18), for $\epsilon=2 p-1$ the operator $M_{\epsilon}$ has exactly one negative eigenvalue and zero is the second eigenvalue. All this together proves lemma II.3.

For the next lemma we define $\epsilon=\frac{2 p-1-\gamma}{\gamma+1}$ and we notice that $1<$ $\epsilon<2 p-1$ for $0<\gamma<p-1$ and $\epsilon<1$ for $\gamma>p-1$.

Lemma II.4. For $0<\gamma<p-1$ the operator $M_{2}$ defined by (2.25) is positive definite. For $\gamma>p-1$ the operator $M_{2}$ is invertible and it has exactly one negative eigenvalue.

Proof. If we define $s(x)=q\left(\frac{x}{\sqrt{\beta}}\right)$ then, in terms of $M_{\epsilon}$ defined by (2.26), (2.25) can be written as $M_{\epsilon} s=\lambda s / \beta$ and the proof follows from lemma II.3. 
Lemmata II.2 and II.4 count the number of negative eigenvalues of $L$ and the next step is to count the number of positive eigenvalues of the symmetric matrix $R\left(\beta_{1}, \beta_{2}\right)$ defined by $(2.15)$.

If $Q_{1}\left(\beta_{1}, \beta_{2}\right)$ is as in (2.11), we see, for instance, that

$$
\frac{\partial Q_{1}\left(\beta_{1}, \beta_{2}\right)}{\partial \beta_{1}}=\int_{\mathbb{R}} u \frac{\partial u}{\partial \beta_{1}} d x .
$$

To prove condition C.3 for the matrix defined in (2.15) we need information about the derivatives $\frac{\partial u}{\partial \beta_{1}} \frac{\partial v}{\partial \beta_{1}}, \frac{\partial u}{\partial \beta_{2}}$ and $\frac{\partial v}{\partial \beta_{2}}$. Differentiating (1.6) implicitly with respect to $\beta_{1}$ at $(\beta, \beta)$ and $u=v$ and denoting $\left(\frac{\partial u}{\partial \beta_{1}}, \frac{\partial v}{\partial \beta_{1}}\right)=(h, k)$ we have

$$
\begin{array}{r}
-h_{x x}+\beta h-((2 p-1)+\gamma) u^{2 p-2} h-\gamma p u^{p-2} k=-u \\
-k_{x x}+\beta k-\gamma p u^{2 p-2} h-((2 p-1)+\gamma(p-1)) u^{2 p-2} k=0
\end{array}
$$

For $\left(\frac{\partial u}{\partial \beta_{2}}, \frac{\partial v}{\partial \beta_{2}}\right)$ a similar system can be obtained. As before, in terms of $r=h+k$ and $q=h-k$ we see that $(2.27)$ decouples into

and

$$
M_{1} r=-r_{x x}+\beta r-(2 p-1)(\gamma+1) u^{2 p-2} r=-u
$$

$$
M_{2} q=-q_{x x}+\beta q-(2 p-1-\gamma) u^{2 p-2} q=-u
$$

In terms of $r$ and $q$ the matrix $R$ defined by (2.15) becomes

$$
R=\frac{1}{2}\left(\begin{array}{cc}
\langle u, r+q\rangle & \langle u, r-q\rangle \\
\langle u, r-q\rangle & \langle u, r+q\rangle
\end{array}\right)
$$

and then

$$
\operatorname{det}(R)=\langle r, u\rangle\langle q, u\rangle \quad \operatorname{tr}(R)=\langle u, r+q\rangle
$$

Lemma II.5. The quantities appearing in $\operatorname{det}(R)$ in (2.30) satisfy:

i) for $\gamma>0$ we have $\langle r, u\rangle>0$;

ii) for $\gamma>p-1$ we have $\langle q, u\rangle>0$; for $\gamma<p-1$ we have $\langle q, u\rangle<0$.

Proof. Differentiating (2.16) with respect to $\beta$ we see that the solution of (2.28) is given by $r=\frac{\partial u_{\beta}}{\partial \beta}$. Therefore,

$$
\langle r, u\rangle=\left\langle\frac{\partial u_{\beta}}{\partial \beta}, u_{\beta}\right\rangle=\frac{1}{2} \frac{d}{d \beta}\left\langle u_{\beta}, u_{\beta}\right\rangle
$$


Moreover, in view of (2.19) we have

$$
\begin{aligned}
\int_{\mathbb{R}^{N}} u_{\beta}^{2}(x) d x & =\left(\frac{\beta}{\gamma+1}\right)^{1 /(p-1)} \int_{\mathbb{R}^{N}} \phi_{0}^{2}(\sqrt{\beta} x) d x \\
& =\frac{\beta^{(3-p) /(2 p-2)}}{(\gamma+1)^{1 /(p-1)}} \int_{\mathbb{R}^{N}} \phi_{0}^{2}(x) d x
\end{aligned}
$$

and then

$$
\frac{d}{d \beta}\left\langle u_{\beta}, u_{\beta}\right\rangle=\frac{(3-p)}{(2 p-2)} \frac{\beta^{(5-3 p) /(2 p-2)}}{(\gamma+1)^{1 /(p-1)}} \int_{\mathbb{R}^{N}} \phi_{0}^{2}(x) d x>0
$$

because $2 \leq p<3$ and this proves $\mathrm{i}$.

To prove ii, using (2.19) we first write (2.29) in terms of $\phi_{0}$ :

$$
-q_{x x}+\beta q+\frac{\beta(\gamma+1-2 p)}{\gamma+1} \phi_{0}^{2 p-2}(\sqrt{\beta} x) q=-\left(\frac{\beta}{\gamma+1}\right)^{1 /(2 p-2)} \phi_{0}(\sqrt{\beta} x)
$$

Next, defining $s(x)=q\left(\frac{x}{\sqrt{\beta}}\right)$ we see that $s(x)$ satisfies

$$
-s_{x x}(x)+s(x)+\frac{(\gamma+1-2 p)}{\gamma+1} \phi_{0}^{2 p-2}(x) s(x)=-\frac{\beta^{(3-2 p) /(2 p-2)}}{(\gamma+1)^{1 /(2 p-2)}} \phi_{0}(x)
$$

and

$$
\int_{\mathbb{R}} q(x) \phi_{0}(\sqrt{\beta} x) d x=\frac{1}{\sqrt{\beta}} \int_{\mathbb{R}} q\left(\frac{x}{\sqrt{\beta}}\right) \phi_{0}(x) d x=\int_{\mathbb{R}} s(x) \phi_{0}(x) d x .
$$

Therefore $\langle q, u\rangle$ and $\left\langle s, \phi_{0}\right\rangle$ have the same sign. Moreover, if we drop the positive factor in front of $\phi_{0}$ in the right hand side of (2.32), the sign of $\left\langle s, \phi_{0}\right\rangle$ does not change. In other words, we can assume that $s$ satisfies

$$
-s_{x x}(x)+s(x)+\frac{(\gamma+1-2 p)}{\gamma+1} \phi_{0}^{2 p-2}(x) s(x)=-\phi_{0}(x)
$$

and we have to analyze the sign of $\left\langle s, \phi_{0}\right\rangle$.

We first consider $\gamma>p-1$. According to lemma II.4, for $\gamma>p-1$ the operator defined by the left hand side of (2.33) is positive definite. Therefore, multiplying (2.33) by $s$ and integrating we get $\left\langle s, \phi_{0}\right\rangle<0$ and this proves the first part of ii.

To prove the second part, we define as before $\epsilon=\frac{2 p-1-\gamma}{\gamma+1}$ and then (2.33) can be written as

$$
M_{\epsilon} s=-s_{x x}+s-\epsilon \phi_{0}^{2 p-2}(x) s=-\phi_{0}(x)
$$


with $1<\epsilon<2 p-1$ because $0<\gamma<p-1$. Since $M_{\epsilon}$ is invertible in that range, the solution $s(\epsilon)$ of (2.34) is well defined and it is smooth in $\epsilon$. Next we show that $\left\langle s(\epsilon), \phi_{0}\right\rangle$ is a decreasing function of $\epsilon$. In fact, defining $z=\frac{\partial s}{\partial \epsilon}$ and differentiating $(2.34)$ with respect to $\epsilon$ we get

$$
M_{\epsilon} z=s \phi_{0}^{2 p-2} \text {. }
$$

Multiplying this last equation by $s$ and integrating we get

$0<\int_{\mathbb{R}} s^{2}(x) \phi_{0}^{2 p-2}(x) d x=\left\langle M_{\epsilon} z, s\right\rangle=\left\langle z, M_{\epsilon} s\right\rangle=\left\langle z,-\phi_{0}\right\rangle=-\frac{d}{d \epsilon}\left\langle s(\epsilon), \phi_{0}\right\rangle$

and then $\left\langle s(\epsilon), \phi_{0}\right\rangle$ is a decreasing function of $\epsilon$. For $\epsilon=2 p-1$, according to lemma II.1, zero belongs to the spectrum of $M_{\epsilon}$. In despite of that, $\phi_{0}$ belongs to the range of $M_{\epsilon}$ because, in view of the same lemma, $\phi_{0}$ is orthogonal to the kernel of $M_{\epsilon}$. In fact, defining

$$
v(x)=v(\beta)(x)=\beta^{1 /(2 p-2)} \phi_{0}(\sqrt{\beta} x)
$$

we see that

Therefore, a solution of

$$
-v_{x x}+\beta v-v^{2 p-1}=0 .
$$

$$
-s_{x x}+s-(2 p-1) \phi_{0}^{2 p-2} s=-\phi_{0}
$$

is

and then

$$
s=\left.\frac{d}{d \beta} v(\beta)\right|_{\beta=1}
$$

$$
\begin{aligned}
\left\langle s, \phi_{0}\right\rangle & =\left\langle\left.\frac{d}{d \beta} v(\beta)\right|_{\beta=1}, \phi_{0}\right\rangle=\frac{1}{2} \frac{d}{d \beta}(\langle v(\beta), v(\beta)\rangle)_{\beta=1} \\
& =\frac{1}{2} \frac{d}{d \beta} \beta^{(3-p) /(2 p-2)} \int_{\mathbb{R}} \phi_{0}^{2}(x) d x>0
\end{aligned}
$$

because $p<3$.

Since $\left\langle s(\epsilon), \phi_{0}\right\rangle$ is decreasing for $1<\epsilon<2 p-1$ and $\left\langle s(2 p-1), \phi_{0}\right\rangle>0$, the lemma is proved.

Proof of theorem I.1 We have to verify conditions C2 and C3. We start with C2. Since, according to lemma II.4, $M_{2}$ is invertible for $0<\gamma \neq p-1$, we conclude that the the kernel of $L$ is given by the kernel of $M_{1}$ and then C2 is a consequence of lemma II.2.

Next we deal with condition C3. According to lemmata II.2 and II.4, for $0<\gamma<p-1$ the operator $L$ has one negative eigenvalue and for $\gamma>p-1$ the operator $L$ has two negative eigenvalues. Moreover, according to lemma 
II.5 and (2.30) in, $R$ has one positive eigenvalue for $0<\gamma<p-1$ and two positive eigenvalues for $\gamma>p-1$. Therefore condition C3 is verified and the theorem is proved.

\section{References}

[1] A. Ambrosetti and E. Colorado, Bound and ground states of coupled nonlinear Schrodinger equations, C.R.Acad.Sci.Paris, Ser I 342 (2006) 453-458.

[2] D. de Figueiredo and O. Lopes, Solitary waves for some nonlinear Schrodinger systems, AHIP Analyse non Linéaire, to appear.

[3] Grillakis,M., Shatah,J and Strauss,W., Stability Theory of Solitary Waves in the Presence of Symmetry, part I, J. Functional Analysis 74,no. 1, 1987,160-197, part II, 94, no. 2, 1990, 308-348.

[4] T.C. Lin and J. Wei, Ground states of N coupled nonlinear Schrodinger equations in $\mathbb{R}^{N}, n \leq 3$, Comm. Math. Phys 225 (2005) 629-653.

[5] T.C. Lin and J. Wei, Ground states of N coupled nonlinear Schrodinger equations in $\mathbb{R}^{N}, n \leq 3$, Comm. Math. Phys., Erratum in press.

[6] L. Maia, E. Montefusco and B. Pellaci, Positive solutions for a weakly coupled nonlinear Schrodinger system, J. Diff. Eq., 229 (2006), 743-767.

[7] D. Pelinovsky and Y. Kivshar, Stability criterion for multicomponent solitary waves, Physical Review E, volume 62, number 6 (2000), 8668-8676.

[8] B. Sirakov, Least Energy Solitary Waves for a System of Nonlinear Schrödinger Equations in $\mathbb{R}^{N}$, Comm. Math. Phys., Volume 271, Number 1, 2007, pg 199-221. 